\title{
LA CONSOMMATION COMPULSIVE DE DROGUES COMME TENTATIVE DE SE SÉPARER D'UNE JOUISSANCE ARCHAÏQUE
}

\author{
Silvia Rivera-Lagarcha, Patrick de Neuter
}

De Boeck Supérieur | «Cahiers de psychologie clinique »

2008/2 $\mathrm{n}^{\circ} 31 \mid$ pages 179 à 198

ISSN 1370-074X

ISBN 9782804157883

Article disponible en ligne à l'adresse :

https://www.cairn.info/revue-cahiers-de-psychologie-clinique-2008-2-page-179.htm

Distribution électronique Cairn.info pour De Boeck Supérieur.

(C) De Boeck Supérieur. Tous droits réservés pour tous pays.

La reproduction ou représentation de cet article, notamment par photocopie, n'est autorisée que dans les limites des conditions générales d'utilisation du site ou, le cas échéant, des conditions générales de la licence souscrite par votre établissement. Toute autre reproduction ou représentation, en tout ou partie, sous quelque forme et de quelque manière que ce soit, est interdite sauf accord préalable et écrit de l'éditeur, en dehors des cas prévus par la législation en vigueur en France. Il est précisé que son stockage dans une base de données est également interdit. 


\title{
LA CONSOMMATION COMPULSIVE DE DROGUES COMME TENTATIVE DE SE SÉPARER D'UNE JOUISSANCE ARCHAÏQUE
}

\author{
Silvia Rivera
}

Patrick De Neuter

Dans l'étude des rapports problématiques aux drogues, la question de la séparation se présente en première instance comme une impossibilité. En effet, lors d'une observation superficielle, les dits « toxicomanes » peuvent apparaitre comme des sujets prêts à se séparer de n'importe quel objet sauf des drogues. Cependant, lors d'une observation plus profonde, aux cours d'entretiens cliniques réalisés en Colombie, en France et en Belgique, expérience clinique doublée d'une recherche systématique à l'aide d'entretiens de recherche, nous avons découvert que cette impossibilité de séparation de ces substances chimiques relève d'une problématique générale beaucoup plus complexe qui dépasse largement cette étroite union matérielle entre le sujet et l'objet chimique. Dans cet article nous allons montrer comment, bien avant leur rencontre avec 
1 Nous allons expliquer ce concept de l'Autre lacanien dans la suite de cet article.

2 Le travail de recherche auquel nous faisons ici référence est présenté de manière plus élargie dans la thèse doctorale « Les consommations de drogues, la jouissance et le lien social ". Développé et soutenue par Silvia Rivera Largacha le 19 décembre 2007 à I'Université Catholique de Louvain, sous la direction des professeurs Philippe Lekeuche et Patrick De Neuter. les drogues, les sujets dits « toxicomanes » éprouvent une difficulté profonde qui concerne leur capacité de se séparer de ce qui les détermine bien avant leur naissance, à savoir, ce que Lacan appelait l'Autre ${ }^{1}$ parental et l'Autre du social. Plus précisément, la plupart de ces sujets semblent avoir été marqués par un sentiment de difficulté et souvent d'impossibilité de produire une séparation par rapport à la jouissance archaïque que Lacan appelle 1'Une-jouissance, jouissance caractéristique des premières modalités de rapport à l'Autre, notamment à l'Autre maternel. De ce fait, ces sujets semblent se perdre progressivement dans la force inouie d'une jouissance sans limite tendant au domaine de l'Un, c'est-à-dire à l'indistinct, à l'excès et finalement à la destruction du sujet. Contrairement aux idées généralement admises, nous en sommes venus à penser que, malgré cette tendance au domaine de l'Un, l'usage compulsif des drogues constitue - aussi et en même temps - un outil dont les sujets se servent pour éviter leur perte définitive dans cette jouissance caractérisant les premières modalités de rapport à l'Autre. Cela constitue, à notre avis, une des spécificités du recours compulsif aux drogues. Il s'agit d'une condition dans laquelle le sujet ne se résigne pas aux alternatives produites par la particularité de sa structure et de sa condition subjective. En effet, ce sujet choisit de chercher dans l'excès des drogues, aussi bien que dans tout ce que ces objets comportent en tant que discours social, un instrument qui pourrait éviter leur chute - et leur perte - définitive dans le domaine de l'Un.

\section{Présentation de la recherche}

Les réflexions que nous allons présenter découlent pour une part importante d'un travail d'observation clinique systématique réalisé en France auprès de huit personnes accueillies dans des Centres spécialisés ayant comme caractéristique commune le travail en ville qui n'inclut pas l'enfermement ${ }^{2}$. Les sujets que nous avons rencontrés témoignent par conséquent d'un désir d'arrêter leur consommation de drogues et de prendre distance par rapport aux différentes sensations obtenues dans la pratique de la consommation compulsive de drogues. Ceci avec tous les paradoxes et toutes les contradictions impliqués 
dans cette décision de renoncer à ces objets qui pendant longtemps ont occupé la place d'une véritable solution pour ces sujets. Lors de notre travail d'observation clinique nous avons utilisé des entretiens de recherche semi-directifs ouverts qui ont été enregistrés et retranscrits et sur lesquels nous avons procédé à des analyses de discours.

Avant de présenter notre travail, il convient encore d'apporter la précision suivante. Bien que nous n'ayons pas centré nos entretiens de recherche sur le diagnostic de la structure de ces sujets, tous ceux que nous avons rencontrés lors de cette enquête en France semblent relever du champ de la névrose. De ce fait, nos propositions valent pour le type de fonctionnement caractéristique de cette structure psychique. Cependant nous pensons pouvoir ajouter que l'utilisation de drogue répond toujours à une nécessité du sujet de dépasser, d'aller audelà des mécanismes de fonctionnement spécifiques de sa structure psychique. Par conséquent, nos observations ouvrent aussi la possibilité de développer la recherche sur l'usage des drogues dans une perspective trans-structurelle : nous faisons l'hypothèse à vérifier dans de nouvelles recherches que notre conclusion principale concerne aussi les sujets pervers et psychotiques.

$\mathrm{Au}$ sujet de cette généralisation de nos conclusions, nous pouvons dire que notre expérience clinique nous apporte certains indices nous menant à penser qu'il est possible de trouver des données similaires dans des conditions individuelles et contextuelles différentes à celles qui ont servi comme basse à cette étude. Bien que les données analysées pour l'élaboration de cet article et de la thèse doctorale dont il est issu, soient réduites à huit sujets, l'enquête originale était constituée par trente personnes dont quatorze d'origine colombienne et vivant dans ce pays. De même, un des auteurs de cet article a continué son travail clinique auprès de consommateurs de drogues même après le recueil et la systématisation des données principales. Ses observations et les conclusions de cette expérience clinique ont servi de point de comparaison pour la lecture et l'analyse des entretiens de recherche. Ces conditions particulières favorisant l'obtention de données diverses et riches, nous ont mené à croire que, malgré les différences culturelles, les variations dans les types de consommation ${ }^{3}$ et même malgré la diversité dans la prééminence des types cliniques ${ }^{4}$ des deux
3 II est évident que de part les conditions de fonctionnement du marché, les produits consommés dans les deux pays sont différents. En Colombie ce sont les produits dérivés de la feuille de coca qui dominent le marché, tandis qu'en France les opiacés et les drogues chimiques sont les produits le plus souvent utilisés.

4 En comparaison avec notre groupe de recherche français, II semblerait que parmi les sujets rencontrés en Colombie, il y avait une prééminence beaucoup plus marquée de personnes ayant une structure psychotique. 
groupes interviewés, il existe des mécanismes communs en ce qui concerne les dynamiques psychiques constituant la base des rapports problématiques des sujets aux drogues.

Nous voudrions dire aussi que l'expérience clinique en Colombie nous a apporté un regard interculturel permettant d'introduire, dans notre recherche, une nouveauté. En Colombie il existe encore beaucoup de groupes indiens ayant des pratiques de construction des liens sociaux impliquant des expériences de consommation de produits psychoactifs naturels. Cette présence réelle d'une certaine «domestication » des effets des psychotropes est présente dans la culture, en tant que référence des effets de solution apportés par ces substances. En effet, très souvent, dans des entretiens cliniques de recherche, cette référence apparait de manière claire et directe lorsque les personnes évoquent leur rapport aux drogues comme une solution à leurs défaillances dans les rapports sociaux. Dans les témoignages des participants français, cette référence est beaucoup plus voilée et se trouve mélangée avec des remords et des intentions d'opposition et de révolte.

\section{L'histoire de Madame C.}

En raison des contraintes imposées par l'écriture de cet article, nous allons proposer des références limitées à une série d'entretiens de recherche cliniques réalisés avec une femme que nous appellerons Madame C. Il s'agit d'une jeune femme de 27 ans, mère d'un petit garçon âgé de deux ans. Depuis un an, elle est rentrée en France après avoir vécu neuf ans dans un autre pays d'Europe occidentale. Pendant les deux dernières années de son séjour dans ce pays, elle a consommé différents produits notamment l'héroïne et le crack. Cette expérience se déroule dans un contexte difficile où elle se trouve confrontée à diverses expériences douloureuses. Dans ces conditions, elle augmente progressivement sa consommation, tout en perdant les stratégies d'autocontrôle sur sa pratique.

À son retour en France, elle prend contact avec une association au sein de laquelle elle débute une thérapie. Ce travail lui permet d'analyser la prise de drogue, non comme un accident dépourvu de sens, mais comme une conséquence directe d'un «état d'âme » non reconnu antérieurement qui s'est révélé 
après deux ans de consommation croissante. Cet « état d'âme » est fait d'une profonde tristesse et d'une grande haine qui s'originent dans les attouchements sexuels qu'elle a subis de la part de son père à partir de l'âge de 8 ans jusqu'au divorce de ses parents survenu vers l'âge de 11 ans.

Madame C. a commencé à prendre de l'héroïne suite au décès de son père. Elle vit cette mort comme la perte de toute possibilité de re-signification des épisodes traumatiques de la rencontre en corps à corps avec lui. Jamais ne seront reconnus par son père les effets traumatiques de cette expérience incestueuse. À l'entendre, cette disparition annule toute possibilité de symboliser l'excès de stimulation vécue de manière prématurée, à travers la vengeance par exemple ou par une reconnaissance des événements de la part du père. Selon Madame $\mathrm{C}$, son père était un homme respecté dont personne ne pouvait soupçonner les dérives, notamment son comportement sexuel à l'encontre de sa fille. En l'absence de cette reconnaissance paternelle, il s'avérait difficile pour cette femme de faire reconnaître sa douleur par l'Autre du social. Elle affirme ainsi : "Les gens le voyaient comme quelqu'un de très, très gentil. Très respectable. Et quand il était avec moi c'était quelqu'un de très, de très violent, méchant. Oui, donc, en fait il n'y a que moi qui a vraiment vu les deux côtés de sa personnalité. "

$\mathrm{Au}$ niveau de la dynamique familiale, la pratique de la drogue s'installe comme une modalité d'expression de son mécontentement et de son mal-être. À travers cette pratique, elle fait une demande, elle réclame une reconnaissance ${ }^{5}$ de sa souffrance. «Je n'en avais rien à foutre des autres, de ma mère, tout le monde me faisait chier, [...]. Et puis, prendre de la drogue, c'était une façon de leur dire que ça n'allait pas. »

Par la pratique d'intoxication, elle essaie de dépasser les lois de la mort tout en réclamant à une figure parentale une resignification de l'expérience vécue. En l'absence du père mort, elle adresse cet appel à sa mère qu'elle considère également responsable de la souffrance qu'elle a subie. À ce propos, elle déclare : «Et... le fait de prendre de la drogue c'était un peu une vengeance pour moi. C'était : regarde maman! Tu as vu? Ça ne va pas! Tu vois ? Là je prends de la drogue, ça veut dire que ça ne va vraiment pas. "

Pour Madame $\mathrm{C}$, l'incapacité de sa mère à découvrir le secret entre son mari et sa fille, de même que son silence face
5 Lacan, J. (1996).

Leçon du 13 mai 59.

Le désir et son interprétation : séminaire 1958-1959, Association freudienne internationale. Paris. Lacan, J. (1966). Les Ecrits, Seuil, Paris. p. 690-691. 
6 Roudinesco, E., Plon, M. (2006). Autre. In Dictionnaire de la psychanalyse. Paris : Fayard. p. 83.

7 Lacan, J. (1966). La chose freudienne ou Sens du retour à Freud en psychanalyse [1955]. In Écrits 1. Livre de poche. Paris : Le Seuil. p. 398-433. au mal-être pourtant évident de sa fille, est ressenti comme une forme de complicité. "Ma mère, je lui en ai voulu parce qu'elle (n') a rien vu. [...] Parce qu'elle (n') était pas là quand j'avais besoin d'elle.»

S'il n'y a pas eu, dans la réalité, de revendication explicite quant à son père, par contre, les problèmes engendrés par la drogue ont permis à Madame $\mathrm{C}$ de s'exprimer haut et fort à l'égard de sa mère : au moment où elle se trouve dans une situation de risque dans le pays étranger où elle résidait, c'est à sa mère qu'elle adresse son appel. Celle-ci arrive juste à temps pour la récupérer et éviter qu'elle perde la garde de son enfant. Cependant, la relation entre les deux femmes reste difficile. Pour Madame C., l'écoute de sa mère est arrivée trop tard.

\section{La fonction de l'Autre : ponctuations pour introduire la place du recours aux drogues dans le rapport du sujet aux objets}

En fonction des entretiens de recherche, nous pouvons affirmer que les personnes qui, comme Madame $\mathrm{C}$, témoignent d'un rapport problématique aux substances psycho-actives, signalent des tensions sociales et familiales qui, par un effet d'accoutumance ou de répression, sont devenues imperceptibles pour leur entourage. Autrement dit, il existe chez ces sujets une difficulté archaïque, dans leur rapport à l'Autre parental et social, qui les conduit à chercher désespérément une solution pour se protéger de cet Autre et s'assurer une place en tant que sujets du désir.

Afin de rendre compte sur le plan psychanalytique de cette difficulté archaïque nous ferons d'abord référence à la notion de l'Autre. Nous envisageons ici cette notion comme désignant l'espace de l'altérité en tant que rapport du sujet «à son entourage, à son désir et à l'objet, dans la perspective d'une détermination inconsciente. » 6

À partir de 1949 Lacan propose l'élaboration de cette notion de l'Autre comme le lieu où se constitue le sujet ${ }^{7}$. De ce fait, le rapport à l'Autre détermine le sujet puisque, contrairement aux identifications imaginaires et spéculaires propres au rap- 
port du sujet à l'autre, le rapport du sujet à cet Autre, avec un A majuscule, est marqué par son impossibilité d'être assimilé par l'intermédiaire de l'identification. Cette impossibilité répond au fait que le sujet est pris dans un ordre absolument extérieur et antérieur à lui-même.

La détermination du sujet par ce grand Autre est identifiée par Lacan, dans un premier temps, lorsqu'il travaille sur la notion du Symbolique d'après la lecture des « Structures élémentaires de la parenté » de Levi-Strauss. Dans cette perspective, Lacan affirme l'existence d'une détermination du sujet par l'ordre Symbolique qu'il désigne comme étant le «lieu de l'Autre».

Dans son interrogation concernant le désir de l'Autre, ce que l'Autre veut par rapport à lui, le sujet se questionne quant à sa propre identité. Notamment en ce qui concerne sa position sexuelle. «Que me veut-il», «que suis-je pour lui ?» sont des questions qui habitent tout enfant. Lacan complexifie encore la notion de l'Autre en signalant que, du fait que le grand Autre a son origine dans une absence, dans un manque fondateur, c'est-à-dire dans ce que Freud appelle «la Chose », il comporte également une part impossible à saisir par le Symbolique, autrement dit, impossible à mettre en mot. Par conséquent, le sujet est condamné à l'impossibilité d'atteindre une connaissance totale de ce que lui veut cet Autre et donc de ce qui le constitue en tant que déterminé par ce dernier. Dès lors le sujet est condamné à vivre une frustration fondamentale : il est littéralement sujet, au sens d'assujetti. Il est soumis à cet Autre et notamment à cette Autre scène comme disait Freud pour désigner l'inconscient.

\section{L'origine de l'Autre dans la Chose}

Arrêtons-nous un instant pour expliciter quelque peu cette origine de l'Autre dans la Chose. Lors des premières expériences de la vie du sujet humain, il y a une précipitation de sensations et d'objets perçus comme un seul objet de satisfaction. Lors de ces expériences, le sujet éprouve une oscillation aléatoire entre le plaisir et le déplaisir. Grâce à l'instabilité des objets, émerge l'activité mentale c'est-à-dire le jugement qui recherche la 
8 Freud S. « Esquisse d'une psychologie scientifique », 1995, repris dans " La naissance de la psychanalyse » PUF, 1956, p. 309-396 production d'un certain équilibre et d'une cohérence des expériences.

En 1895, dans son travail «Esquisse d'une psychologie scientifique », Freud utilisa le concept de das Ding, terme que Lacan reprend sous la dénomination de 'la Chose'. Das Ding chez Freud $^{8}$, la Chose chez Lacan, est la trace laissée chez le sujet par l'objet perdu avant que le sujet ne soit passé par le processus marquant la perte irrémédiable de celui-ci. Das Ding est donc essentiellement une absence, un manque qui est à l'origine de ce que Freud appelle les motivations éthiques de l'humain et de cette dépendance qui le lie à tout jamais à l'Autre. Une fois la Chose perdue, apparaît une disparité irrémédiable entre le sujet et l'objet. La jouissance qui correspond à la Chose, jouissance archaïque, est irrémédiablement perdue. L'absence, qui est le lieu de la Chose, est dès lors visée par le sujet, de façon permanente, comme espace de retour. Il y revient par l'articulation de la voix qui est d'abord un cri et ultérieurement une parole. Ainsi, voix, parole et langage se constituent comme voies incontournables pour les tentatives répétées du sujet essayant de retrouver cette jouissance à jamais perdue.

C'est par le biais du langage qu'il s'adresse à l'Autre lorsqu'il lui formule une demande. Si la parole, comme instrument pour s'adresser à l'Autre, garde en soi la trace de son origine dans la Chose, l'Autre, ayant la même provenance, en garde également l'empreinte. En ce sens, l'enseignement tardif de Lacan révèle une nouvelle perspective sur l'Autre. Celui-ci est explicité en tant qu'Autre circonscrit dans l'ordre symbolique. L'Autre serait le détenteur de la Loi qui sépare le sujet de la Chose mais, en même temps, il est la jouissance de cette Chose, inatteignable parce qu'interdite. Ainsi, bien que l'Autre soit prioritairement dans l'ordre symbolique, il y a quelque chose en sa base qui appartient toujours à la dimension du Réel, autrement dit à la dimension de l'inatteignable, de l'indicible, de l'inimaginable et du non symbolisable. Dans cette dimension la Chose trouve sa place. Ainsi, la figure de l'Autre plonge aussi ses racines dans le terrain mouvant de la jouissance en tant qu'archaïque et dépourvue de limites. 


\section{La notion de jouissance}

Il est important de développer maintenant une analyse du rapport entre l'Autre, qui détermine le sujet, et la dimension de la jouissance. Cette analyse nous permettra de rassembler les outils conceptuels nécessaires à la compréhension de ce que nous avons repéré, dans la pratique compulsive des drogues, concernant le rapport du sujet à l'Autre.

Disons tout d'abord que le terme de « jouissance » fait référence aux formes de rapport que le sujet établit aux objets. Comme nous venons de l'évoquer, ces modalités de satisfaction ou de frustration sont déterminées par un premier rapport : le rapport à la Chose. Ce dernier se caractérise par le dépôt dans la mémoire de traces d'un état de plénitude suivi d'une séparation douloureuse par rapport à cet état. Dès lors, cet état de plénitude première est perdu dans la préhistoire du psychisme et demeure comme une trace qui devient la base de toute relation ultérieure aux objets du désir. Grâce à ces traces, le sujet garde sur lui la nostalgie de l'objet perdu dont l'existence, en tant qu'inatteignable, appartient au Réel. La jouissance a son origine dans ce rapport primordial à la Chose.

Cependant, il existe une partie de cette jouissance prioritairement réelle qui est traversée par les effets de la castration, de la découverte de la différence des sexes, de la rencontre du langage, autrement dit par l'entrée dans le Symbolique. Lacan a nommé la jouissance appartenant prioritairement au Réel «Une-jouissance », puisqu'elle est propre au régime du « faire Un » avec la Chose. La « jouissance phallique » ou « jouissance sexuelle » est le résultat de cette jouissance lorsqu'elle traverse et est traversée par le Symbolique. Dans son séminaire sur l'Angoisse, Lacan décrit le processus de transformation de la jouissance dès lors qu'elle passe par la séparation progressive du sujet par rapport à cet Autre archaïque qui est, en première instance, représenté par l'Autre parental. Ainsi, il montre que la vacillation du sujet face au désir de l'Autre parental, est un passage nécessaire pour que la jouissance, en tant qu'Une-jouissance, jouissance essentielle et jouissance masturbatoire soit transformée en jouissance sexuelle phallique autrement dit en jouissance du corps de l'Autre sexe ${ }^{9}$. Ceci ouvre les possibilités de la naissance du sujet au champ du désir. Le désir est dans cette perspective le résultat d'une
9 Par ce dernier terme, Lacan fait référence à cette jouissance qui, du fait qu'elle est traversée par les effets de la castration, est marquée par les exigences de l'Autre comme limites de la satisfaction pulsionnelle totale. La jouissance du corps de l'Autre est la jouissance bordée de limites grâce auxquelles le sujet reconnaît la marque des différences, différence des sexes et différence des générations, etc., de ce fait, elle ouvre des possibilités d'établissement du désir. 
acceptation de l'impossibilité d'atteindre une satisfaction pulsionnelle totale.

La catégorie de la jouissance rassemble ainsi une multiplicité de thématiques et de questionnements concernant les rapports entre les sujets, en tant que sujets de la pulsion. Par ailleurs, ces sujets possèdent un corps, voire sont possédés par un corps qui tend toujours, d'une part, à la satisfaction la plus plénière possible et d'autre part à l'évitement du déplaisir. Cette catégorie de la jouissance dévoile en outre les complexes dynamiques constituant le processus de satisfaction pulsionnelle que Freud avait déjà reconnu comme fonctionnant selon une tendance à l'au-delà du principe de plaisir, c'est-à-dire à une tendance à la pulsion de mort.

Ceci étant explicité, nous pouvons nous poser la question de savoir quelle est, quelles sont la ou les modalité(s) de jouissance que l'on retrouve chez les sujets consommateurs compulsifs de drogues. Autrement dit: "Que cherche un sujet jouissant et souffrant de son rapport aux drogues ?» Chercherait-il, à travers l'expérience toxique, le rétablissement de cette relation primordiale avec la Chose, relation toute empreinte de l'Une-jouissance ? Ou, au contraire, chercherait-il la construction de nouvelles modalités de rapport avec des objets divers du monde ? C'est-à-dire la construction de rapports d'objet tendant à la jouissance du corps de l'Autre sexe?

Suite à l' analyse de nos entretiens de recherche, doublée de notre expérience clinique en France, et en Colombie, nous pensons pouvoir dire qu'il s'agit d'une tendance du sujet à osciller entre ces deux modalités de jouissance. La particularité de cette oscillation consiste en ceci qu'elle se présente toujours sous une forme paradoxale où les limites et les différentiations entre les deux types de jouissance tendent à s'effacer. Mais comment expliquer ce phénomène ?

\section{La jouissance dans le recours aux drogues}

L'analyse de la fonction des drogues, dans la confrontation aux différentes modalités de jouissance, comporte une difficulté particulière de fait de la diversité des expériences qui peuvent être vécues par le sujet dans la rencontre de ces subs- 
tances. Dans les paragraphes suivants, nous essayerons d'expliquer à la lumière de la catégorie de jouissance, quels sont les différents moments qui marquent les diverses modalités du rapport au toxique. En effet, la pratique des drogues peut être envisagée en premier lieu, comme la restitution d'un état primitif perdu, état associé à des expériences de grand plaisir. Dans cette première perspective, la rencontre avec la drogue pourrait être décrite en référence à la définition du plaisir apportée par Freud dans « Au-delà du principe de plaisir ». On dira qu'il s'agit là d'une tentative visant à diminuer l'excès d'excitation dans l'appareil psychique. En général, la première rencontre avec la drogue est motivée par l'espoir de trouver un quelque chose afin de traiter un mal-être ressenti consciemment ou inconsciemment.

Par exemple dans le cas de Madame C, la drogue est assimilée à « un deuil ». Mme C. utilise cette expression pour signifier deux éléments cruciaux de sa rencontre avec le toxique. Elle recourt à la drogue au moment décisif de sa vie où la mort de son père survient par une malheureuse coïncidence le jour même de la naissance de son fils. Peu de temps après, le père de son fils décède lui aussi, victime d'une crise cardiaque. Sur cette série d'épreuves mêlées à la présence de la drogue, elle s'explique ainsi : "Et je (n') ai jamais pleuré quand j'ai pris de la drogue. Je (n') ai jamais pleuré».

Le deuxième élément significatif de l'emploi de ce terme du « deuil » fait référence à l'absence du produit. En effet, l'absence de produit qui, dans un premier temps, a apaisé la douleur, réveille maintenant chez elle un nouveau « deuil». Elle se trouve dans une situation de fragilité. Elle doit faire face à ses anciens traumatismes auxquels s'ajoute le traumatisme actuel engendré par l'absence de produit. Par rapport à ce deuxième "deuil », celui de la drogue, elle déclare : " [...] j'y pense tous les jours. C'est comme quand on a perdu quelqu'un de sa famille, [...]. Oui, oui. Je suis en train de faire un deuil.».

L'expérience du sevrage est douloureuse. L'abandon de la drogue n'est pas ce qu'elle avait imaginé : une affaire de quelques jours pour se débarrasser du manque physique. Elle découvre dans ce processus une douleur psychique qu'elle avait cru avoir éteinte ou au moins contrôlée quand elle avait fait le choix de se servir de la drogue comme solution. C'est à cette 
10 Jacques, J-P. (1999). Pour en finir avec les toxicomanies. Psychanalyse et pourvoyance légalisée des drogues. Collection : Oxalis. De Boeck.

Bruxelles.

11 Terme utilisé pour faire référence à l'expérience de satisfaction pulsionnelle extrême retrouvé dans certaines formes d'imprégnation des drogues sur le corps.

12 Le Poulichet, S. (1987). Toxicomanies et psychanalyse. Paris, PUF.

13 Ceci est une caractéristique de tout objet d'investissement libidinal mais, dans le cas des drogues, elle se révèle de manière très radicale, notamment dans les cas où existe une pratique compulsive. dimension de la douleur précédant et accompagnant la répétition compulsive de la pratique des drogues que Jean-Pierre Jacques ${ }^{10}$ fait référence lorsqu'il utilise la catégorie d'appétence. Cette catégorie montre que cette tentative d'anesthésier la douleur et d'atteindre une détente psychique est liée, dès le début, aux expériences traumatiques éprouvées avant et durant la rencontre avec la drogue.

Dans le recours aux drogues, la douleur, qui est à l'origine de l'appétence, se révèle dans l'acte même de l'imprégnation de la substance sur le corps. À ce propos, Philippe Lekeuche explique que même dans l'expérience du « flash » ${ }^{11}$, il existe un caractère surprenant et traumatique du fait de l'excès de l'expérience.

Le retour de ce qui devait être annulé par les effets de la drogue se produit en raison de deux facteurs. Le premier est la double nature du pharmakon que Sylvie Le Poulichet a bien mis en évidence dans son travail « Toxicomanies et psychanalyse ${ }^{12}$. En reprenant le mythe de Theuth récité dans Phèdre, Le Poulichet aborde la question de la toxicomanie comme actualisation de la figure ambiguë du pharmakon grec. Comme on le sait, ce dernier est un élément dont la nature ambiguë peut en faire un remède ou un poison notamment de par sa capacité à produire des « excès ». En nous référant à cette catégorie grecque, nous pouvons affirmer que, du fait de l'instabilité des effets des drogues (incarnations actuelles du pharmakon), il existe une tendance de ces entités chimiques à produire des effets opposés à ceux qui sont recherchés par celui qui s'en sert ${ }^{13}$. En résumé, ce qui a été cherché comme remède peut devenir un poison.

Le second facteur, produisant le retour de la douleur dans la pratique des drogues, est le caractère insistant du contenu du trauma qui serait à l'origine de la douleur qui est la source de l'appétence. Ce contenu se révèle par l'érosion des effets de la drogue, érosion entendue comme chute des illusions construites autour des effets du chimique. Nous avons pu observer qu'une fois que ces illusions s'amenuisent, l'effet recherché par le sujet n'opère plus.

Contrairement à ce que l'on pourrait croire, l'érosion des effets du toxique n'est pas seulement une conséquence de l'épuisement des effets du chimique dans l'organisme. Il s'agit 
aussi d'une transformation du rapport du sujet à la substance chimique et cela peut coïncider ou non avec l'épuisement des effets chimiques sur l'organisme. Les expériences dites de 'mauvais voyage', se traduisant par des vomissements lors des premières prises d'héroïne, les visions atterrantes produites par l'utilisation de certains hallucinogènes, et d'autres sensations déplaisantes ou effrayantes provoquées par différents types de psychotropes, sont les éléments qui peuvent servir de voie de retour pour la douleur psychique associée au traumatisme qui fut à l'origine de cette tentative de solution. Ces phénomènes sont des érosions psychiques, et non chimiques, des effets des drogues. Les répercussions secondaires de la pratique de consommation telles que le rejet social, l'échec professionnel ou scolaire, ou les effets physiopathologiques ${ }^{14}$, peuvent être eux aussi à l'origine d'une érosion des effets psychiques des drogues. Enfin, les événements qui, d'une certaine façon, exigent une transformation quant à la reconnaissance symbolique du sujet, peuvent également produire ce phénomène. Par exemple, la naissance d'un enfant, la mort d'un proche ou une rencontre amoureuse. Dans le cas de Madame C c'est le risque de perdre la garde de son enfant qui produit une érosion des effets du toxique. C'est à ce moment-là qu'il se produit chez elle une transformation quant à la reconnaissance symbolique d'elle-même. Face au risque de perdre son enfant elle est appelée à occuper la place de mère qu'elle n'avait pas encore vraiment occupée.

Nous voyons donc qu'il existe en général deux éléments marquant le rapport du sujet au toxique. D'une part, le caractère insistant du contenu du trauma qui est à l'origine d'une douleur, ravivée par l'expérience de l'érosion des effets des drogues. D'autre part, la recherche d'une anesthésie s'opposant aux manifestations du déplaisir. Nous avons pu observer dans notre clinique comme dans nos entretiens de recherche que ces éléments se présentent simultanément, même si le sujet reconnaît rarement cette production synchronique. Face à l'illusion de la toute-puissance du chimique dans la recherche d'une protection contre la douleur, survient - en même temps - la désillusion de cet espoir. Par la rencontre de ces deux éléments contraires et paradoxalement simultanés, le sujet est confronté à la jouissance en tant qu'espace illimité de l'Une-jouissance. Ainsi, en proie à l'oscillation radicale entre
14 Des maladies infectieuses, des problèmes de circulation, des maladies dentaires et d'autres pathologies fréquentes parmi les usagers de drogues. 
15 Freud, S. (1981). Au-delà du principe de plaisir [1920]. In Essais de psychanalyse. Paris : Payot. p. 43-115.

16 Une recherche souvent confondue de l'extérieur avec la recherche d'une sensation de plaisir sans limites.

17 En effet, dans tous les témoignages nous retrouvons une progression des sujets vers l'incapacité de retrouver du plaisir dans les expériences, même dans le cas des expériences d'intoxication. anesthésie et douleur, le sujet semble perdre les repères qui lui permettraient d'échapper au destin fatal de l'Une-jouissance, c'est-à-dire Jouissance de l'Un, plaisir et douleur sans limite. C'est seulement dans la reconnaissance et la reconstitution d'images et de significations opposées entre ces deux épreuves, à savoir le plaisir et la douleur, que le sujet pourra poser des limites à l'Une-jouissance. Notre expérience nous indique que les entretiens cliniques peuvent apporter cela au sujet.

Cette confusion entre plaisir et douleur, émergeant dans la consommation des drogues, nous renvoie aux élaborations concernant l'intrication permanente entre ces deux dimensions de l'être. Dans «Au-delà du principe de plaisir » ${ }^{15}$, Freud soutient que la recherche du plaisir, c'est-à-dire de l'évitement de tout excès d'excitation dans l'appareil psychique, vise le retour à un état antérieur à la vie. Dans le chemin conduisant au domaine d'un au-delà du principe de plaisir, les représentations de la douleur, qui ont marqué l'émergence de la vie chez le petit humain, se multiplient.

La consommation de drogues, comme pratique visant l'apaisement de la douleur, chercherait aussi à provoquer la précipitation de douleurs extrêmes correspondant à ce moment inaugural de la vie où le petit humain éprouve, de manière saisissante, les premières rencontres avec le plaisir et le déplaisir. C'est pour cela que la rencontre postérieure à cette oscillation entre expériences extrêmes, n'est pas un accident, mais un phénomène recherché par les sujets qui témoignent d'un rapport problématique aux drogues.

Dans la continuité des propositions de Freud sur le plaisir et le déplaisir, Lacan relit la théorie d' " Au-delà du principe de plaisir » en se servant de la philosophie du droit de Hegel. À partir de ce travail, il propose une théorie du rapport du sujet à ces expériences organiques qui, de par l'effet des interventions du langage venues de l'Autre, deviennent des expériences du corps. De ce fait, en suivant le modèle freudien, Lacan explique le plaisir non pas comme un phénomène illimité mais, au contraire, comme une limite s'imposant à l'excès que comporte la constitution même de la libido chez l'humain.

Ainsi, nous pouvons conclure que, s'il existe un sujet qui cherche de manière insistante l'anesthésie de la douleur ${ }^{16}$ tout en perdant de plus en plus sa capacité de ressentir plaisir ${ }^{17}$, 
c'est parce qu'il y a quelque chose en lui qui échoue dans l'organisation de la libido. En ce sens, contrairement à ce qui peut être affirmé par les modèles cognitivo-comportementaux, tout comme par certains modèles psychanalytiques, la consommation compulsive de drogues ne serait pas seulement un essai pour échapper à la douleur, mais aussi et surtout une tentative pour donner une nouvelle signification à celle-ci par l'érosion des effets du chimique. Cette érosion laisse apparaitre l'insistance d'une sensation de malêtre dépourvue de bornes. Cette douleur est étouffée, de manière transitoire, par la répétition de la prise. Cependant l'insistance de la douleur précédant la prise et existant à l'intérieur même du rapport aux drogues, rapproche de plus en plus le sujet du royaume de la jouissance sans limites, auquel il tentait d'échapper dans un premier moment avec la drogue. Par conséquent, à un moment ou à un autre, se présente au sujet la nécessité, de chercher une autre interprétation de sa douleur et par là, de son plaisir.

À travers l'érosion du recours aux drogues existerait une possibilité naissante pour le sujet de fixer des bornes plus précises à l'Une-jouissance archaïque afin d'accéder de manière plus permanente aux privilèges de la jouissance du corps de l'Autre sexe. Cependant, il ne s'agit pas là d'un parcours assuré car le sujet risque de ne pas arriver à trouver une sortie de la douleur, du traumatisme premier produisant de l'appétence, ni de la douleur des traumatismes secondaires produits par les effets de la pratique des drogues. Dans ce sens, il risque de perdre progressivement toute possibilité de prendre distance par rapport à "l'Une-jouissance » tout en voyant s'effacer la différence qui sépare cette modalité de jouissance de la "Jouissance du corps de l'Autre sexe ».

C'est dans la conjoncture privilégiée de l'érosion du recours aux drogues qu'une intervention thérapeutique peut apporter au sujet une issue à l'oscillation infernale entre les extrêmes de l'excès de l'Une-jouissance. Il ne s'agit pas cependant de privilégier une précipitation par le sevrage à tout prix. En étant conséquents avec ce que nous venons d'expliquer au sujet de la dimension de jouissance existant dans l'épuisement des effets réels du toxique, il est clair que le déplaisir du sevrage radical peut être aussi une incarnation de l'Une- jouissance menaçant le sujet. Dans ce sens il serait beaucoup plus adéquat d'apporter au sujet un sevrage graduel dans lequel la 
confrontation aux excès et aux traumatismes secondaires produits par les effets de la pratique des drogues sont diminués. On favorisera ainsi une analyse de l'expérience de rapport problématique aux drogues. Cette analyse serait réalisée à partir d'une distance par rapport à "l'Une-jouissance » qui comporte le déplaisir sans bornes produit par le sevrage radical. Le clinicien peut aider le sujet à articuler, dans les deux sens de ce mot, les traumatismes secondaires produits par les effets de la pratique des drogues. Dans ce processus, il serait possible de reconnaître le caractère de solution qui a été, dans un premier temps, assigné par le sujet aux effets des drogues. C'est par cette voie qu'il serait possible de reconnaitre l'échec de l'espoir construit autour des effets du chimique, pour enfin reconstituer et analyser les traumatismes premiers qui ont été à la base de cet espoir.

\section{Jouissance de l'Autre}

À ce point de notre réflexion, s'impose à nous la nécessité d'expliquer pourquoi ces sujets ont d'abord rencontré cette difficulté ou cette impossibilité de mettre des limites à l'Unejouissance archaïque ; pourquoi sont-ils affectés par cette difficulté profonde de s'en séparer.

Pour ce faire, il nous faut à présent montrer comment le sujet construit des limites à la Jouissance afin d'identifier les accidents dans ce processus dont ont souffert ceux qui ont connu une difficulté radicale dans le rapport aux drogues. À ce propos, nous dirons que le corps du sujet, en tant que sujet de la parole et du désir, est livré aux signifiants et aux contraintes de son inscription dans la dimension de la demande. Il est sujet du désir en tant que désirant et en tant qu'objet du désir de l'Autre parental. En effet, dans la production du symptôme, dans le plaisir ou la douleur, le sujet est joui, puisqu'il fait jouir l'Autre - c'est du moins ce qu'il imagine en même temps qu'il jouit lui-même. Cela est possible car le sens de son symptôme, caché pour lui-même, se trouve dans le trésor des signifiants de l'Autre. Ainsi, la production du symptôme est une forme de jouissance pour le sujet et pour l'Autre dont le sens reste voilé pour le premier. On peut l'évoquer en parlant de la forme de jouissance fantasmée et horri- 
fiante d'une Mère ou d'un Père primitif qui, comme le Père du mythe de «Totem et Tabou », jouirait sans limites ${ }^{18}$. D'où la nécessité pour le sujet que l'Autre soit lui aussi traversé par la Loi humaine fondamentale. Sans quoi, il ne peut ni les transmettre à l'enfant, ni non plus sauvegarder ce dernier de la destruction par sa jouissance illimitée et sa férocité.

À écouter tous les sujets de notre recherche, il semblerait qu'ils aient fait, d'une façon ou d'une autre, l'expérience d'une confrontation à un Autre parental qui se présenterait comme mal traversé par la Loi fondamentale. On comprend que, dans ces conditions, le sujet se serait senti menacé de périr dans la jouissance de la mère ou du père. Si ces deux figures ou l'une d'entre elles ne sont pas elles-mêmes marquées par les limites de la castration, une cohabitation des jouissances multiples n'est pas possible pour le sujet.

Dans le cas de Madame $C$, c'est le père qui n'incarne pas l'interdit de l'inceste ; cette Loi symbolique fondamentale, qui devrait permettre à sa fille d'éprouver une reconnaissance de sa désirabilité, tout en restant libre de l'éventualité d'une rencontre amoureuse en dehors du cadre familial ${ }^{19}$.

La consommation des drogues a souvent été confondue, même par des modèles psychanalytiques, avec une tentative itérative pour atteindre la jouissance du corps, l'Une-jouissance. Toutefois, à partir des données recueillies lors de notre travail clinique et dans notre recherche systématique, il nous semble que le recours aux drogues cherche plutôt à empêcher la chute du sujet dans cette jouissance sans limites, mortifère et désubjectivante.

Dans tous les cas que nous avons rencontrés, le sujet avait eu affaire, comme Madame C, avec une figure de l'Autre faisant de sa jouissance la Loi pour l'enfant. Or, là où il n'y pas de Loi, il n'y a pas de désir, disait Lacan. Et l'on observe en effet que le désir s'étiole lorsque plus rien ne fait obstacle à sa totale réalisation. Mais la fonction de la Loi est aussi de rendre possible la construction du sujet en séparant sa jouissance de celle de l'Autre, en mettant des limites aux caprices de l'Autre et à l'angoisse qu'ils induisent fatalement chez le sujet. L'absence partielle ou totale de cette Loi, dans le lieu de l'Autre, est toujours catastrophique pour le sujet. L'Autre, non traversé par la Loi, est à l'origine d'une figure effrayante et mortifère
18 Freud S., « Totem

et Tabou » (1912-1913)

Payot, 1965.

19 Comme toute jeune fille, Madame C a dû se confronter à ses propres tentatives de séduction du père. Mais, elle a dû le faire dans une situation défavorable, puisque son père n'a pas incarné l'interdit que sa fille réclamait par ses tentatives de séduction. D'où sa difficulté de se débarrasser des images incestueuses se répétant dans ses rêves et ses souvenirs diurnes. La massivité et l'excès qui comporte la répétition de ces images révèle l'existence d'une jouissance illimitée insistante chez Madame $\mathrm{C}$, qui semble résister à toute possibilité de séparation de cette femme par rapport à la tendance archaïque de I'Une-jouissance. Cela peut expliquer l'énorme difficulté de femme pour trouver et pour se reconnaître un désir propre ; surtout en ce qui concerne le champ du désir érotique envers des hommes. En effet, le rapport amoureux exigerait de sa part une ouverture vers la modalité de la jouissance du corps de l'Autre sexe, mais, en même temps celui-ci évoquerait la rencontre avec cette jouissance massive et archaïque imposée par le père. 
20 Dans le cas de Madame C, il s'agirait d'une mère qui, par son indifférence face aux rapports incestueux entre son mari et sa fille, semble faciliter ce rapport tout en mettant sa fille à la place qu'ellemême devrait occuper. Cette identification radicale entre mère et fille dans le domaine de la jouissance finit par être une menace de destruction pour la jeune fille qui essaie de se débarrasser des traces laissées sur elle par les abus de son père et de l'indifférence, quelque part complaisante, de sa mère par rapport à cette situation. qui s'approprie la jouissance du sujet et, par là, le fait disparaître.

Dans la pratique itérative du recours à la drogue chez tous les 'participants' à notre recherche, nous avons repéré, non pas une recherche d'une jouissance sans limites mais, au contraire, une tentative d'instaurer une distance par rapport à cette «Unejouissance archaïque » qui les reliait encore à l'Autre en tant qu'émargeant du domaine de la Chose.

Pour ces sujets, comme pour tout autre, les modalités de référence à l'Autre du social s'enracinent dans la manière dont le rapport à l'Autre de l'enfance a été résolu. Et nous avons en effet retrouvé, chez ces personnes, l'angoisse face à la menace provenant de la jouissance effroyable de la Mère ${ }^{20}$, ou de la jouissance d'un Père ayant les caractéristiques du Père Primitif de « Totem et tabou ».

La progression de la pratique itérative des drogues indique bien que c'est l'effet de l'érosion des effets du produit, le ratage de la drogue, qui en vient à produire chez le sujet une mise-en-manque. Cette rencontre avec la dimension du manque correspond à un manque d'efficacité du produit dans la recherche d'effets d'apaisement par le sujet. Il s'agit d'un manque imaginaire et symbolique qui, en première instance, ne permet pas au sujet de mettre des bornes au réel de l'Unejouissance. Cependant, dans une autre perspective, cette miseen-manque s'établit comme un essai pour assurer l'existence de cette castration qui a été mal transmise par les Autres de l'enfance. Le sujet souffre en quelque sorte d'un manque du manque, autrement dit, d'une difficulté de séparation d'avec la Chose, d'avec l'Une-jouissance et d'avec un Autre mal traversé par la Loi. Dans ce contexte l'érosion des effets de maîtrise de l'angoisse produits, dans un premier temps, par la drogue, marque l'apparition d'une angoisse secondaire. Celle-ci semblerait plus maîtrisable que l'angoisse informe et débordante qui surgit de la confrontation à un Autre qui n'assure pas une juste distance avec la Chose.

Il nous semble que le recours aux drogues, même sous la forme d'une compulsion, n'est pas, comme certains cliniciens l'ont affirmé, la recherche d'une jouissance sans Autre. Nous avons été amenés à penser au contraire que l'Autre était bien présent dans l'acte de prise de drogue. Il est présent dans la 
tentative du sujet d'échapper à sa jouissance, qui se présente à lui comme totalement ou partiellement dépourvue de limites, autrement dit comme une source d'angoisse. Il est présent aussi dans la tentative du sujet de réclamer de la part de l'Autre l'assurance d'une reconnaissance de la marque de la Loi sur lui-même. Une demande aussi de transmission de cette Loi comme héritage qui pourrait assurer enfin la fixation et la mobilité de son désir ainsi qu'une juste distance par rapport à la Chose.

Cependant, de par la nature instable du pharmakon, et de par l'insistance du trauma qui s'impose à travers la manifestation du symptôme que la drogue tente de masquer, la tentative est régulièrement vouée à l'échec. Par conséquent, la stratégie du recours aux drogues finit par souligner la difficulté du sujet à se séparer, à se couper, à se castrer, de l'Une-jouissance de et avec l'Autre.

Nous voulons conclure cette réflexion par une question qui reste ouverte pour nous en ce qui concerne le rapport entre le sujet qui souffre et jouit de son rapport aux drogues et la figure de l'Autre, notamment en tant qu'Autre parental. Comment rendre compte que pour ces sujets l'Autre soit une figure appartenant prioritairement à une dimension de la Chose ? Dans le cas de Madame C., l'abus sexuel se présente comme point critique de l'impossibilité d'instauration de la Loi pour le sujet et pour l'Autre. Cependant, dans d'autres cas, cela est moins clair. Une recherche ultérieure devrait éclairer les particularités du chemin des sujets qui recourent aux drogues comme solution à une difficulté psychique profonde qui n'affecte pas qu'eux.

LACAN, J., « Leçon du 13 mai 59. Le désir et son interprétation : séminaire 1958-1959 », Association freudienne internationale, Paris, 1996.

LACAN, J., Les Écrits, Le Seuil, Paris, 1966, p. 690-691.

RoudinesCO, E. et M. Plon, « Autre », in Dictionnaire de la psychanalyse, Fayard, Paris,2006, p. 83.

LACAN, J., « La chose freudienne ou Sens du retour à Freud en psychanalyse », in Écrits 1, Livre de poch, Le Seuil, Paris, [1955] 1966, p. 398-433.

FREUD S., «Esquisse d'une psychologie scientifique », 1995 ; repris dans La naissance de la psychanalyse, PUF, 1956, p. 309-396

JACQUeS, J.-P., Pour en finir avec les toxicomanies. Psychanalyse et pourvoyance légalisée des drogues, De Boeck, coll. « Oxalis », Bruxelles, 1999. 
LE Poulichet, S., Toxicomanies et psychanalyse, Paris, PUF, 1987.

FREUD, S., «Au-delà du principe de plaisir », in Essais de psychanalyse, Paris, Payot, (1920) 1981, p. 43-115.

FREUD, S., Totem et Tabou, Payot, (1912-1913) 1965.

$\overline{\text { Résumé On considère souvent que la consommation compul- }}$ sive de drogue vise à obtenir une jouissance sans limites, écho d'une jouissance infinie, jouissance dite par Lacan « jouissance de l'Un » ou encore «Une-Jouissance ». Cette jouissance est supposée exister dans la toute petite enfance avant que les Lois fondamentales humanisantes ne soient transmises au sujet. Sur base d'une large expérience clinique de l'un d'entre eux et d'une quinzaine d'entretiens de recherche approfondis, les auteurs soutiennent que cette recherche de l'Une-jouissance se double d'une tentative souvent méconnue de se séparer de cette même jouissance et ainsi que du rapport incestueux à la Chose (en termes freudiens) à l'Autre parental (en termes lacaniens). Ils soutiennent ainsi que la drogue, comme le pharmakon des grecs, doit être considérée dans sa double valeur : poison tout autant que solution au malaise.

Mots-clés toxicomanie, drogue, jouissance, autre, séparation, castration, das Ding, la Chose.

Summary It is often considered that the compulsive consumption of a drug aims at obtaining a pleasure without limits, echo of an infinite pleasure called by Lacan jouissance of One or "One-jouissance". This pleasure is supposed to exist in the early childhood before the humanizing fundamental Laws have been transmitted to the subject. Based on a large clinical experience of one of the authors and on eight deeper interviews of research, the authors explain that this research of the One-jouissance is produced simultaneously with the attempt, often ignored, to separate from this same jouissance and as well from the incestuous relationship with the Thing (in freudian terms) with the parental Other (in lacanian terms). They also explain that a drug, the pharmakon of the Greeks, must be considered in its double value: a poison as much as a solution to a malaise.

Keywords drug-addiction, drug, pleasure, other, separation, castration, das Ding, the Thing. 